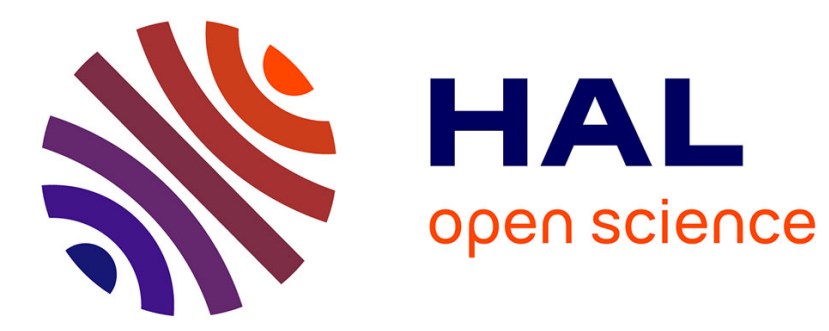

\title{
Spectrométrie neutronique de temps de vol par intercorrélation à l'aide d'un accélérateur comme source de neutrons
}

\author{
E. Descroix, R. Billerey, J. Depraz, M. Rotival, H. Duquesne, A.P. Schmitt
}

\section{To cite this version:}

E. Descroix, R. Billerey, J. Depraz, M. Rotival, H. Duquesne, et al.. Spectrométrie neutronique de temps de vol par intercorrélation à l'aide d'un accélérateur comme source de neutrons. Revue de Physique Appliquée, 1974, 9 (5), pp.939-945. 10.1051/rphysap:0197400905093900 . jpa-00243859

HAL Id: jpa-00243859

https://hal.science/jpa-00243859

Submitted on 1 Jan 1974

HAL is a multi-disciplinary open access archive for the deposit and dissemination of scientific research documents, whether they are published or not. The documents may come from teaching and research institutions in France or abroad, or from public or private research centers.
L'archive ouverte pluridisciplinaire HAL, est destinée au dépôt et à la diffusion de documents scientifiques de niveau recherche, publiés ou non, émanant des établissements d'enseignement et de recherche français ou étrangers, des laboratoires publics ou privés. 
Classification

Physics Abstracts

0.679

\title{
SPECTROMÉTRIE NEUTRONIQUE DE TEMPS DE VOL PAR INTERCORRÉLATION A L'AIDE D'UN ACCÉLÉRATEUR COMME SOURCE DE NEUTRONS
}

\author{
E. DESCROIX, R. BILLEREY, J. DEPRAZ \\ Institut de Physique Nucléaire, Université Claude Bernard-Lyon I \\ et Institut National de Physique Nucléaire et de Physique des Particules \\ 43, bd du 11-Novembre-1918, 69621 Villeurbanne, France
}

et

\author{
M. ROTIVAL, H. DUQUESNE, A. P. SCHMITT \\ C. E. N. Cadarache, 13-Saint-Paul-lez-Durance, France
}

(Reçu le 7 février 1974, révisé le 25 mars 1974)

\begin{abstract}
Résumé. - La méthode d'intercorrélation est appliquée à la spectrométrie neutronique par temps de vol à l'aide d'un accélérateur de $400 \mathrm{keV}$ comme source de neutrons; son domaine de validité par rapport à la méthode classique est précisé. Deux spectres neutroniques de types très différents sont réalisés comme expériences-tests : un spectre monotone de transmission à travers un verre au $\mathrm{Li}$ et un spectre de diffusion élastique sur $\mathrm{W}$.
\end{abstract}

Abstract. - The intercorrelation method is applied to time-of-flight neutron spectrometry, neutrons being produced by a $400 \mathrm{keV}$ accelerator. Its domain of validity is determined and compared with that of the classical method. Two neutron spectra of very different kinds are measured as a test experiment : the first one is a monotonic transmission spectrum through a Li-glass, the other is a spectrum of elastic scattering from $\mathrm{W}$.

1. Introduction. - Les méthodes statistiques d'intercorrélation ont commencé à être employées pour mesurer des réactivités et pour étudier le bruit d'un réacteur [1], [2].

Jusqu'ici, les premières expériences-tests ont été effectuées sur des réacteurs pourvus de "choppers" convenables [3], [4], [5] ; elles ont montré clairement qu'en présence de bruit de fond important, l'amélioration sur la méthode classique récurrente pouvait être importante, ce que prévoient un certain nombre d'études théoriques [6], [7], [8].

Nous avons utilisé ces mêmes méthodes avec un petit accélérateur comme source de neutrons, notamment pour la spectrométrie neutronique par temps de vol [9], [10], [11], [12].

Dans une première partie, nous donnerons le principe de la méthode et la précision statistique obtenue par rapport à la méthode récurrente conventionnelle, puis nous décrirons deux expériences-tests de spectrométrie de temps de vol effectuées par cette méthode à l'aide d'un petit accélérateur de $400 \mathrm{keV}$ :

a) La transmission des neutrons à travers un verre au $\mathrm{Li}$ et la mesure du nombre de noyaux $\mathrm{Li}^{6}$ par $\mathrm{cm}^{3}$. b) La diffusion élastique de neutrons sur du tungstène naturel.

2. Principe (Fig. 1). - Soit un faisceau de neutrons pulsés suivant un code binaire cyclique $X(t)$ généré par

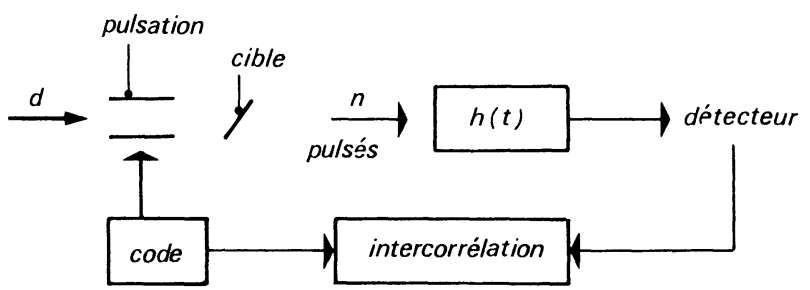

Fig. 1. - Détermination du spectre de temps de vol $h(t)$ par intercorrélation.

un registre à décalages dont le bouclage permet la séquence de longueur optimale. Si $n$ est le nombre d'étages du registre, le nombre d'éléments du code est $N=2^{n}-1$ [13], [14], [15]. $X(t)$ prend les valeurs - 1 ou +1 et peut changer d'états, tous les $\Delta t$; il y a $\frac{N+1}{2}$ états +1 et $\frac{N-1}{2}$ états -1 , par période, 
$T_{0}=N \Delta t$. La valeur -1 correspond à l'absence de neutrons, + 1 à la présence de neutrons.

Si $I(t)$ est l'intensité du faisceau de neutrons en régime continu, l'intensité de neutrons pulsés sur la base de vol sera:

$$
\frac{1}{2}(X(t)+1) I(t)
$$

Ce faisceau pulsé est envoyé sur un système linéaire constitué d'un massif ralentisseur, d'un échantillon et d'une base de vol ; on appelle $h_{0}(t)$ la réponse impulsionnelle du système, c'est le spectre de temps de vol.

La détection des événements en bout de base de vol est faite avec une efficacité $\varepsilon_{0}$ qui dépend du temps de vol. Ces informations sont analysées en temps par un dispositif fonctionnant en multi-échelle de largeur de canal $\Delta t$ égale à la période d'horloge du code de pulsation pour une excursion de $N$ canaux. On prend pour durée d'expérience un nombre entier de période de code $T=(\beta+1) T_{0}, \beta$ entier.

La période $T_{0}$ du code doit être choisie de sorte que $h_{0}\left(T_{0}\right)=0$. Prenons pour unité de temps la largeur $\Delta t$ d'un canal. Soit $Y_{0}(k)$ le nombre de neutrons arrivés en bout de base de vol dans les intervalles de temps

$$
\left[(k-1) \Delta t+\alpha T_{0}, k \Delta t+\alpha T_{0}\right]
$$

avec $k \in[1, N]$ et $\alpha \in[0, \beta]$. Alors

$$
\begin{aligned}
Y_{0}(k)= & \frac{1}{2} \sum_{\alpha=0}^{\beta} \sum_{k^{\prime}=1}^{N} I\left(k-k^{\prime}+\alpha N\right) \times \\
& \times\left[X\left(k-k^{\prime}+\alpha N\right)+1\right] h_{0}\left(k^{\prime}\right)+b_{0}(k)
\end{aligned}
$$

avec $I(k)=$ nombre de neutrons émis pendant l'intervalle $(k-1) \Delta t, k \Delta t ; h_{0}(k)$ représente la probabilité qu'a un neutron d'avoir un temps de vol compris entre $(k-1) \Delta t$ et $k \Delta t ; b_{0}(k)=$ nombre d'informations de bruit de fond arrivées sur le détecteur.

Dans le canal $k$ de l'analyseur le nombre d'informations détectées et stockées pendant $T$ sera donc:

$$
\begin{array}{rl}
Y(k)=\frac{1}{2} \sum_{\alpha=0}^{\beta} \sum_{k^{\prime}=1}^{N} & I\left(k-k^{\prime}+\alpha N\right) \times \\
& \times\left[X\left(k-k^{\prime}\right)+1\right] h\left(k^{\prime}\right)+b(k)
\end{array}
$$

$h(k)=\varepsilon_{0}(k) h_{0}(k)$ représente le spectre de vol expérimental, c'est-à-dire la probabilité qu'a un neutron détecté d'avoir un temps de vol compris entre $(k-1) \Delta t$ et $k \Delta t, b(k)$ est le bruit de fond dans le canal $k$.

La méthode d'intercorrélation consiste à effectuer le calcul

$$
\Gamma_{X Y}(m)=\sum_{k=1}^{N} X(k) Y(k+m) .
$$

En effet, on peut montrer que cette fonction d'intercorrélation est, en valeur moyenne proportionnelle au spectre expérimental $h(k)$. Il est ensuite facile de remonter au spectre vrai à partir de la courbe d'efficacité du détecteur $\varepsilon_{0}(k)$

$$
E\left\{\Gamma_{X Y}(m)\right\}=\sum_{k=1}^{N} X(k) E\{Y(k+m)\}
$$

avec

$$
\begin{aligned}
& E\{Y(k+m)\}= \\
& \quad=\frac{1}{2}(\beta+1) \bar{I} \sum_{k^{\prime}=1}^{N}\left[X\left(k+m-k^{\prime}\right)+1\right] h\left(k^{\prime}\right)+\bar{b}
\end{aligned}
$$

$\bar{I}$ est le nombre moyen de neutrons émis en régime continu pendant $\Delta t ; \bar{b}$ est le bruit de fond moyen par canal (pour la durée de l'expérience)

$$
\begin{aligned}
& E\left\{\Gamma_{X Y}(m)\right\}= \\
& =\frac{1}{2}(\beta+1) \bar{I} \sum_{k^{\prime}=1}^{N} h\left(k^{\prime}\right) \sum_{k=1}^{N} X(k) X\left(k+m-k^{\prime}\right)+ \\
& \quad+\frac{1}{2}(\beta+1) \bar{I} \sum_{k^{\prime}=1}^{N} h\left(k^{\prime}\right)+\bar{b} .
\end{aligned}
$$

En effet le code est construit de telle sorte que

$$
\sum_{k=1}^{N} X(k)=1
$$

De plus, les séquences de longueurs maximales obtenues à partir du registre à décalage ont une fonction d'autocorrélation [14] :

$$
\begin{aligned}
\Gamma_{X X}(m) & =\frac{1}{N} \sum_{k=1}^{N} X(k) X(k+m) \\
& =1 \text { si } m=0 \\
& =-\frac{1}{N} \text { ailleurs }
\end{aligned}
$$

Ce qui permet d'écrire :

$$
E\left\{\Gamma_{X Y}(m)\right\}=\frac{1}{2}(\beta+1) \bar{I}(N+1) h(m)+\bar{b} .
$$

Soit

$$
I_{0}=\frac{1}{2}(\beta+1) \bar{I}(N+1)
$$

le nombre total de neutrons pulsés arrivés sur le détecteur et $b^{\prime}=N \bar{b}$ le nombre d'informations de bruit incorrélé détecté

$$
E\left\{\Gamma_{X Y}(m)\right\}=I_{0} h(m)+\frac{b^{\prime}}{N}
$$

$I_{0} h(m)$ est le nombre de neutrons détectés en temps de vol compris entre $(m-1) \Delta t$ et $m \Delta t$.

Le terme correspondant au bruit incorrélé $b^{\prime} / N$ est difficilement estimable, si ce n'est par une expérience annexe, c'est pourquoi on a intérêt à utiliser des codes aussi longs que possible, de sorte que le terme de bruit soit rendu négligeable. Le dispositif de stockage nous limite dans le choix de la longueur du code (2 047 éléments). Cette limite n'existe plus avec l'utilisation d'un corrélateur en temps réel.

3. Précision statistique sur $\boldsymbol{\Gamma}_{X Y}(\boldsymbol{m})$. $-X(t)$ est un signal certain, les quantités $Y(k+m)$ dans (5) sont indépendantes et poissoniennes, c'est-à-dire :

$$
\sigma^{2}\{Y(k+m)\}=E\{Y(k+m\}
$$


alors :

$$
\begin{aligned}
& \sigma^{2}\left\{\Gamma_{X Y}(m)\right\}=\sum_{k=1}^{N} X^{2}(k) E\{Y(k+m)\} \\
& \sigma^{2}\left\{\Gamma_{X Y}(m)\right\}= \\
& =\frac{1}{2}(\beta+1) \bar{I} \sum_{k=1}^{N} \sum_{k^{\prime}=1}^{N}\left[X\left(k+m-k^{\prime}\right)+1\right] \times \\
& \quad \times h\left(k^{\prime}\right)+N \bar{b} \\
& =(\beta+1)(N+1) \frac{\bar{I}}{2} \sum_{k^{\prime}=1}^{N} h\left(k^{\prime}\right)+N \bar{b}
\end{aligned}
$$

ou avec la notation ( 9 bis $)$ :

$$
\sigma^{2}\left\{\Gamma_{X Y}(m)\right\}=I_{0} \sum_{k^{\prime}=1}^{N} h\left(k^{\prime}\right)+N \bar{b}
$$

et

$$
\sum_{k^{\prime}} h\left(k^{\prime}\right)=\sum_{k^{\prime}} \varepsilon_{0}\left(k^{\prime}\right) h_{0}\left(k^{\prime}\right)
$$

est la probabilité qu'a un neutron de temps de vol quelconque d'être détecté en bout de base de vol, par suite

$$
\sigma^{2}\left\{\Gamma_{X Y}(m)\right\}=I^{\prime}+b^{\prime}
$$

où $I^{\prime}$ représente le nombre total de neutrons corrélés détectés et $b^{\prime}$ le nombre total d'informations de bruit non corrélé détecté. En résumé la variance de la fonction d'intercorrélation $\Gamma_{X Y}(m)$ ne dépend pas du canal et est égale au nombre total d'informations détectées pendant la durée de la mesure $T$.

4. Comparaison des précisions statistiques. - Avant de comparer les précisions statistiques de la méthode de corrélation et de la méthode classique, nous poserons les hypothèses suivantes :

- même faisceau moyen en régime continu pour les deux méthodes,

- même période de répétition et même temps d'expérience,

- la durée $\Delta t$ de la bouffée en méthode classique est égale au retard incrémental de la méthode de corrélation,

- enfin, même bruit moyen par canal.

Justifions la validité de cette dernière hypothèse. Dans les expériences de diffusion et de transmission que nous décrivons plus loin les détecteurs étaient placés dans une pièce voisine de celle de l'accélérateur avec une séparation de plus de deux mètres de béton. Ainsi le bruit ambiant en présence et en l'absence du faisceau était très peu différent dans la pièce des détecteurs et expérimentalement cette hypothèse est assez bien vérifiée.

Le nombre moyen d'informations stockées dans le canal $k$ pendant la durée $T$ de la mesure en méthode classique est

$$
\begin{array}{r}
E\{Y(k)\}=\bar{I}(\beta+1) \sum_{k^{\prime}=1}^{N} X\left(k-k^{\prime}\right) h\left(k^{\prime}\right)+\bar{b} \\
=\bar{I}(\beta+1) h(k)+\bar{b}
\end{array}
$$

puisque

$X(n)=1$ si $n=\lambda N, \quad \lambda$ entier,$\quad X(n)=0$ ailleurs.

La variance pour le canal $k$ est

$$
\sigma^{2}\{Y(k)\}=E\{Y(k)\} .
$$

Pour tirer la valeur du spectre de temps de vol expérimental $h(k)$, il est nécessaire tant en méthode classique qu'en corrélation d'effectuer une mesure annexe donnant une estimation du bruit afin d'obtenir les quantités

$$
\begin{gathered}
E\{Y(k)\}^{\prime}=E\{Y(k)\}-\bar{b} \\
E\left\{\Gamma_{X Y}(k)\right\}^{\prime}=E\left\{\Gamma_{X Y}(k)\right\}-\bar{b} .
\end{gathered}
$$

De ce fait on majore la variance d'une quantité $b$, d'où les expressions :

$$
\begin{aligned}
& \quad\left[\frac{\sigma\{Y(k)\}^{\prime}}{E\{Y(k)\}^{\prime}}\right]_{\text {classique }}^{2}=\frac{\bar{I}(\beta+1) h(k)+2 \bar{b}}{[\bar{I}(\beta+1) h(k)]^{2}} \\
& {\left[\frac{\sigma\left\{\Gamma_{X Y}(k)\right\}^{\prime}}{E\left\{\Gamma_{X Y}(k)\right\}^{\prime}}\right]_{\text {correlation }}^{2}=} \\
& =\frac{\bar{I}}{\frac{2}{2}(\beta+1)(N+1) \sum_{k^{\prime}=1}^{N} h\left(k^{\prime}\right)+(N+1) \bar{b}} \\
& {\left[\frac{\bar{I}}{2}(\beta+1)(N+1) h(k)\right]^{2}}
\end{aligned}
$$

Posons

$$
\bar{S}=\frac{\bar{I}(\beta+1) \sum_{k^{\prime}} h(k)}{N}
$$

nombre moyen d'informations corrélées par canal en méthode classique, et

$$
S_{k}=\bar{I}(\beta+1) h(k)
$$

nombre d'informations corrélées détectées dans le canal $k$ en méthode classique

$$
R^{2}=\frac{(\sigma / E)_{\text {correlation }}^{2}}{(\sigma / E)_{\text {classique }}^{2}}=4 \frac{N \bar{S} / 2+\bar{b}}{\left(S_{k}+2 \bar{b}\right)} \frac{1}{N+1}
$$

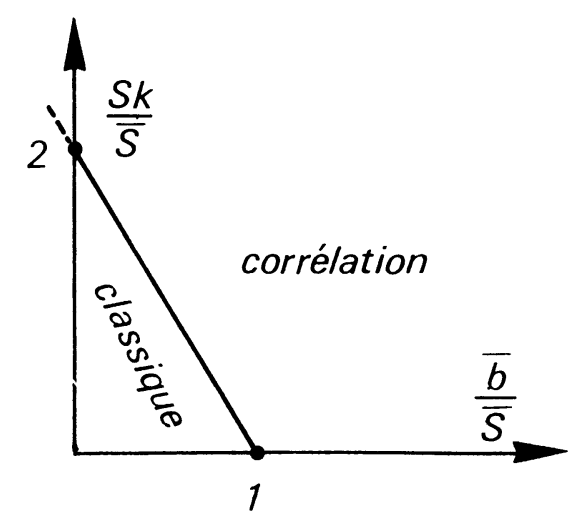

Fig. 2. - Domaine de validité de la méthode classique et de la méthode de corrélation pour le canal $k$ du spectre en fonction du bruit moyen par canal $(\bar{b})$, du signal moyen par canal $(\bar{S})$ et du signal dans le canal $k\left(S_{k}\right)$. (Les grandeurs sont exprimées en méthode classique.) 
La méthode de corrélation est donc supérieure à la méthode classique si

$$
R^{2}<1 \quad \text { c'est-à-dire } 2<\frac{S_{k}}{\bar{S}}+2 \frac{\bar{b}}{\bar{S}} .
$$

La corrélation est meilleure dans les parties hautes des spectres dans tous les cas, dès que $S_{k} / \bar{S}>2$. Pour les parties basses sa supériorité intervient lorsque le rapport $\vec{b} / \bar{S}$ (de la méthode classique) est supérieur à 1 (Fig. 2).

5. Dispositif expérimental. - 5.1 ProduCtion DES NEUTRONS. - Pour tester la méthode d'intercorrélation, de brèves bouffées de neutrons d'énergie comprises entre 4 et $100 \mathrm{eV}$ sont requises. Des neutrons de $14 \mathrm{MeV}$ sont produits par l'intermédiaire de la réaction $D(t, n) \propto$ avec un accélérateur Sames de $400 \mathrm{keV}$. La fluence pour un faisceau de $400 \mu \mathrm{A}$ est de $4 \times 10^{10}$ neutrons par seconde dans les $4 \pi$. Les deutons de $250 \mathrm{keV}$ sont pulsés selon un code binaire cyclique à $50 \%$ par un système déflecteur à onde progressive à 16 plaques. L'impulsion de commande est retardée d'une plaque à la suivante d'un temps égal au temps de vol du faisceau entre deux plaques pour améliorer sensiblement les temps de montée et de descente. L'impulsion de commande est obtenue à partir d'un code fourni par un générateur Hewlett Packard qui est envoyé dans un amplificateur capable de délivrer des impulsions de $300 \mathrm{~V}$ sous une impédance de $100 \Omega$ (impédance de la ligne à retard).

La bonne marche de la pulsation peut être vérifiée d'une part, en examinant le courant de faisceau sur un porte-cible adapté (Fig. 3), d'autre part, en déterminant

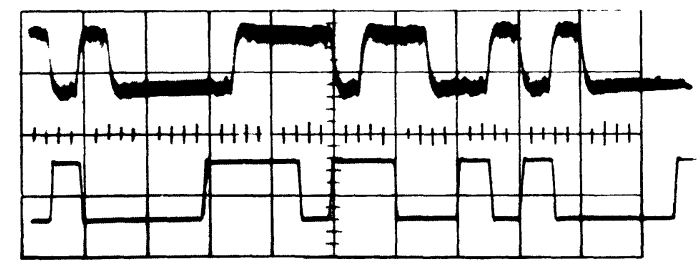

FIG. 3. - Courant de faisceau pulsé sur la cible de tritium et code de pulsation.

la fonction d'intercorrélation du code et du faisceau de neutrons de $14 \mathrm{MeV}$ en bout de la base de vol. Cette fonction d'intercorrélation (Fig. 4) est une fonction triangle analogue à la fonction d'autocorrélation du code. Cette mesure a été réalisée au moyen de détecteurs constitués de $4 \mathrm{PM} \mathrm{NaI}\left(\mathrm{T}_{1}\right)\left({ }^{1}\right)$ associés autour d'un disque de $B^{10}$ et polyéthylène précédé d'un autre disque de $\mathrm{CB}^{4}$ qui joue le rôle de ralentisseur et permet ainsi d'augmenter l'efficacité aux neutrons rapides.

(1) Poussot, R., Pelicier, H., Communication privée, C. E. N. Cadarache.

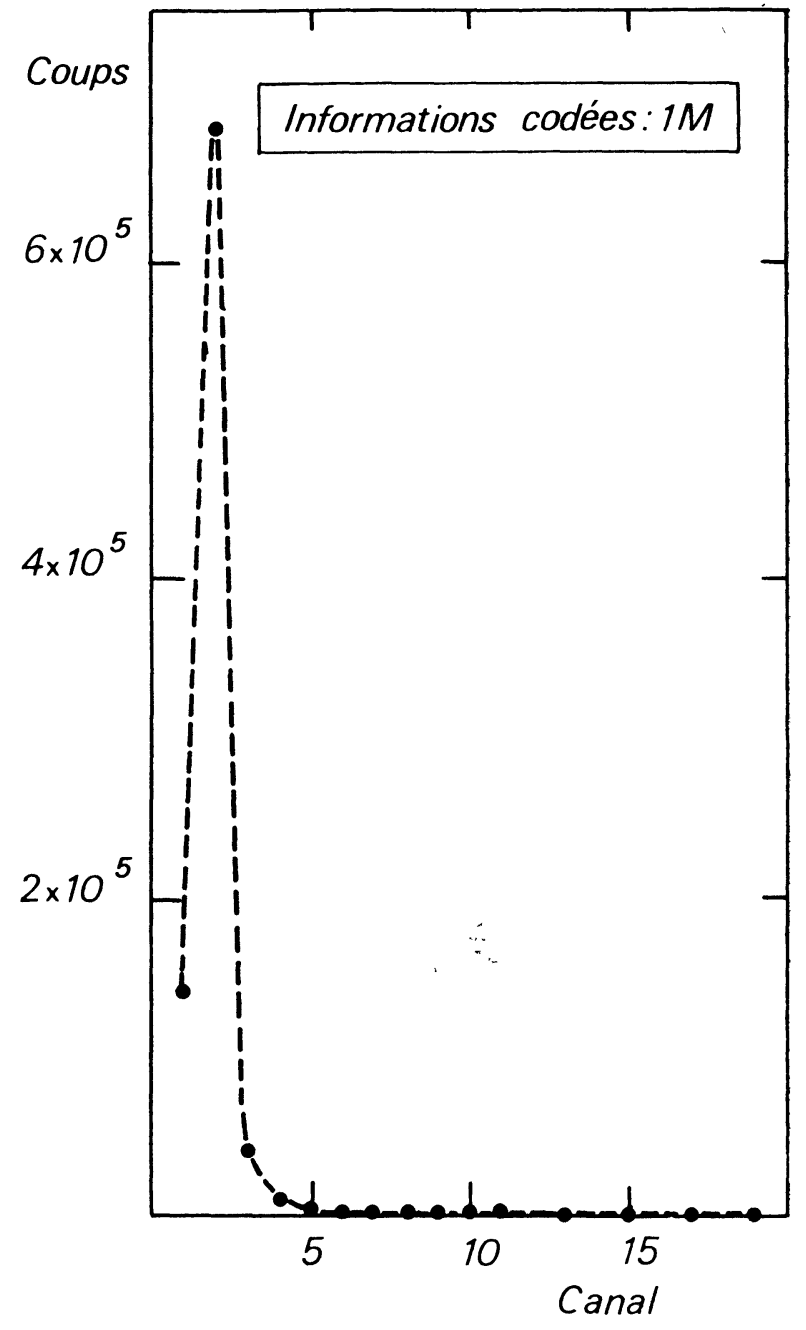

FIG. 4. - Spectre d'intercorrélation des neutrons de $14 \mathrm{MeV}$ pulsés en bout de base de vol et du code de pulsation.

5.2 RALENTISSEMENT DES NEUTRONS. - La cible tritiée $\left(850 \mu \mathrm{g} / \mathrm{cm}^{3}\right)$ est entourée de briques d' $\mathrm{U}^{238} \mathrm{de}$ $4 \mathrm{~cm}$ d'épaisseur dans le but de multiplier le nombre de neutrons $\left({ }^{2}\right)$ et également de dégrader le spectre des neutrons rapides. Le ralentissement est obtenu par $4 \mathrm{~cm}$ de polyéthylène [16]. Autour de ce massif, est placée une feuille de cadmium pour arrêter les neutrons d'énergie inférieure à $0,4 \mathrm{eV}$. Nous avons vérifié que dans ces conditions, pour un retard incrémental $\Delta t=3,2 \mu \mathrm{s}$, le codage existe toujours pour les neutrons lents sortant du massif. La fonction d'intercorrélation code-neutrons ralentis (Fig. 5) détectés à la sortie du massif par une chambre à fission CFU 7 entourée d'indium est en effet une fonction triangle, légèrement élargie par suite du temps de ralentissement et de sa dispersion.

5.3 DÉTECTION DES NeUtRons LENTS. - La base de vol longue de $13 \mathrm{~m}$ aboutit à un collimateur de $80 \mathrm{~cm}$

(2) BRICA, M., Communication privée SESR/ESPCI, C.E.N. Cadarache. 


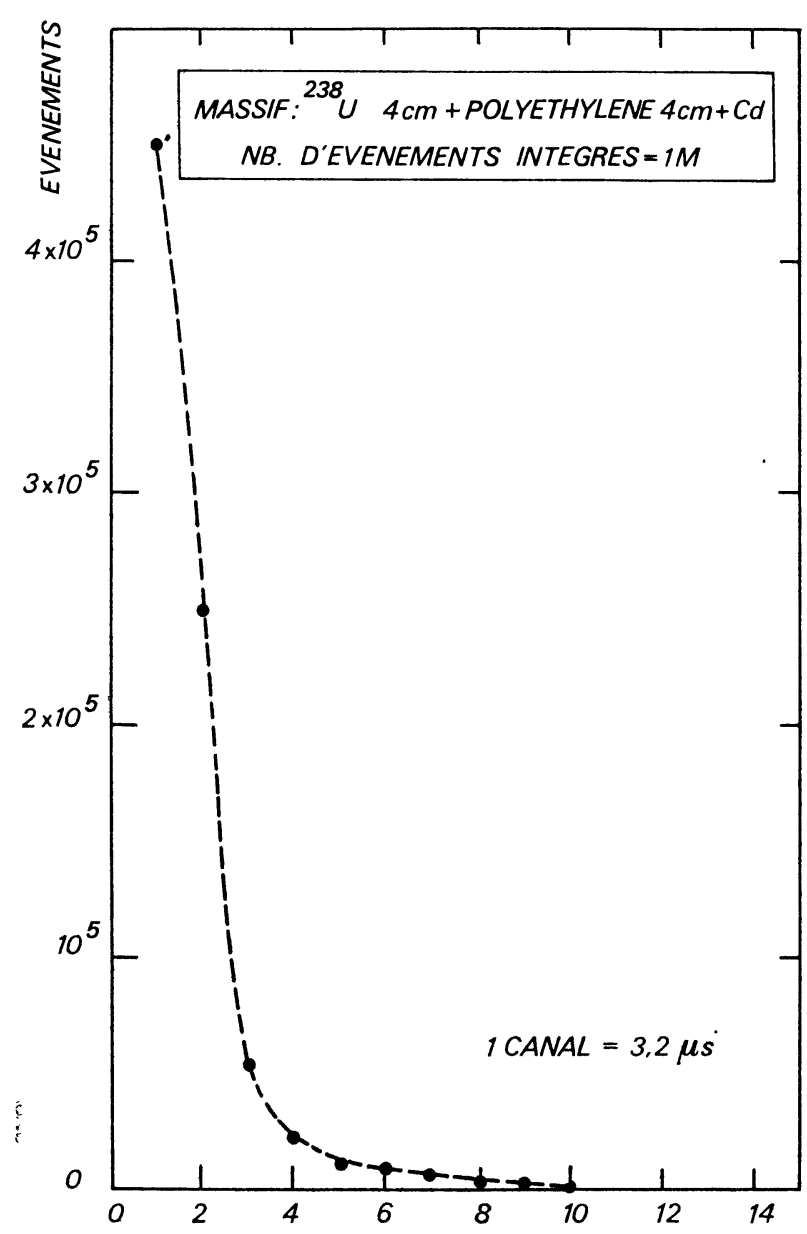

Fig. 5. - Spectre d'intercorrélation des neutrons à la sortie du massif ralentisseur et du code de pulsation (réponse en temps du massif).

de long en carbonate de lithium et se termine par un massif de protection en plomb où l'on peut placer un ou plusieurs détecteurs suivant le type de manipulations.

Pour la détection des neutrons lents, un verre scintillateur KG 2L à faible bruit de fond enrichi en $\mathrm{Li}^{6}$ a été choisi. Il présente une forte efficacité aux neutrons lents et une faible efficacité aux neutrons rapides ; il est pratiquement insensible aux $\gamma$ d'énergie inférieure à 1,2 MeV [17], [18], [19].

5.4 EleCTRONIQUE. - La chaîne électronique est classique ; les informations arrivent sur la voie analyse d'un codeur HC 25 qui pilote également le générateur de code avec un retard incrémental de 3,2 $\mu$ s (Fig. 6). Les informations codées sont stockées dans un BM 96. Le calcul de la fonction d'intercorrélation est fait en temps différé sur ordinateur CDC 6600 . Il nécessite le stockage des informations dans un nombre de canaux égal au nombre $N$ d'éléments du code. La remise à zéro du balayage du codeur HC 25 admet un temps mort ; on est alors obligé d'utiliser comme zone de balayage 2 fois la longueur du code, d'où la présence du diviseur par 2 pour les impulsions de synchro. Ainsi, on peut arrêter le balayage quelques canaux avant la fin de la

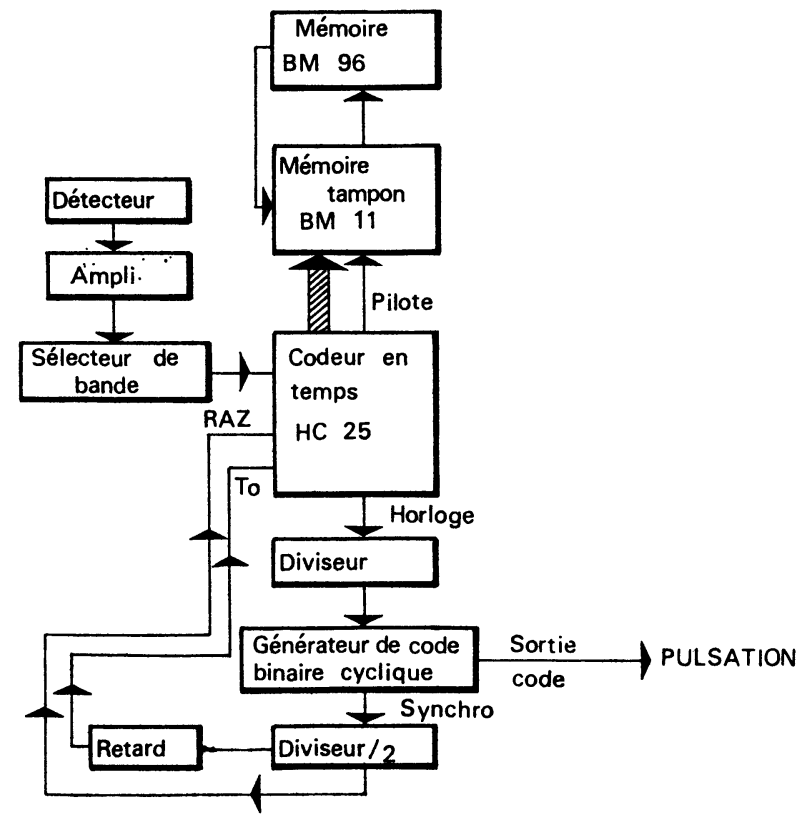

Fig. 6. - Synoptique du codage en temps des informations du détecteur.

$2^{\mathrm{e}}$ période de l'impulsion, le redémarrage $T_{0}$ arrivant avec un retard suffisant pour permettre cette remise à zéro du HC 25.

6. Résultats. - Comme test de la méthode d'intercorrélation, nous avons étudié deux spectres neutroniques de types très différents ; un spectre monotone de transmission d'une part et un spectre de diffusion élastique d'autre part.

6.1 SPECTRE DE TRANSMISSION. - Il s'agit de mesurer le facteur de transmission d'un verre NE 905 enrichi au $\mathrm{Li}^{6}$ de $1 \mathrm{~cm}$ d'épaisseur et de $4,4 \mathrm{~cm}$ de diamètre. Après avoir déterminé la réponse en énergie du massif ralentisseur, le nombre de neutrons transmis (Fig. 7) est mesuré en opérant en une bonne géométrie, c'est-à-dire en plaçant le verre NE 905 au milieu de la base de vol dans un petit collimateur. Pour déterminer le bruit, le verre est remplacé par des absorbants (W et $\mathrm{Ag}$ ) qui ont des raies d'absorption très importantes dans la zone de 4 à $100 \mathrm{eV}$. C'est dans les canaux correspondant à ces raies d'absorption que l'on détermine le bruit moyen. Connaissant la section efficace totale de $\mathrm{Li}^{6}$ [20] nous avons mesuré le nombre de noyaux de $\mathrm{Li}^{6}$ de ce verre. Le résultat obtenu est

$$
14,6 \times 10^{21} \text { atomes } / \mathrm{cm}^{3}
$$

à $\pm 4 \%$. Cette expérience a nécessité $50 \mathrm{~h}$ de faisceau. Le rapport signal sur bruit en méthode conventionnelle était de 1 .

La même mesure a été faite par une équipe de l'accélérateur linéaire de Saclay avec le même verre, par la méthode conventionnelle ; leur résultat est de $\left({ }^{3}\right)$ $14,94 \times 10^{21}$ atomes $/ \mathrm{cm}^{3}$ à $0,6 \%$.

(3) Derien, M., Communication privée, C. E. N. Saclay (1973). 


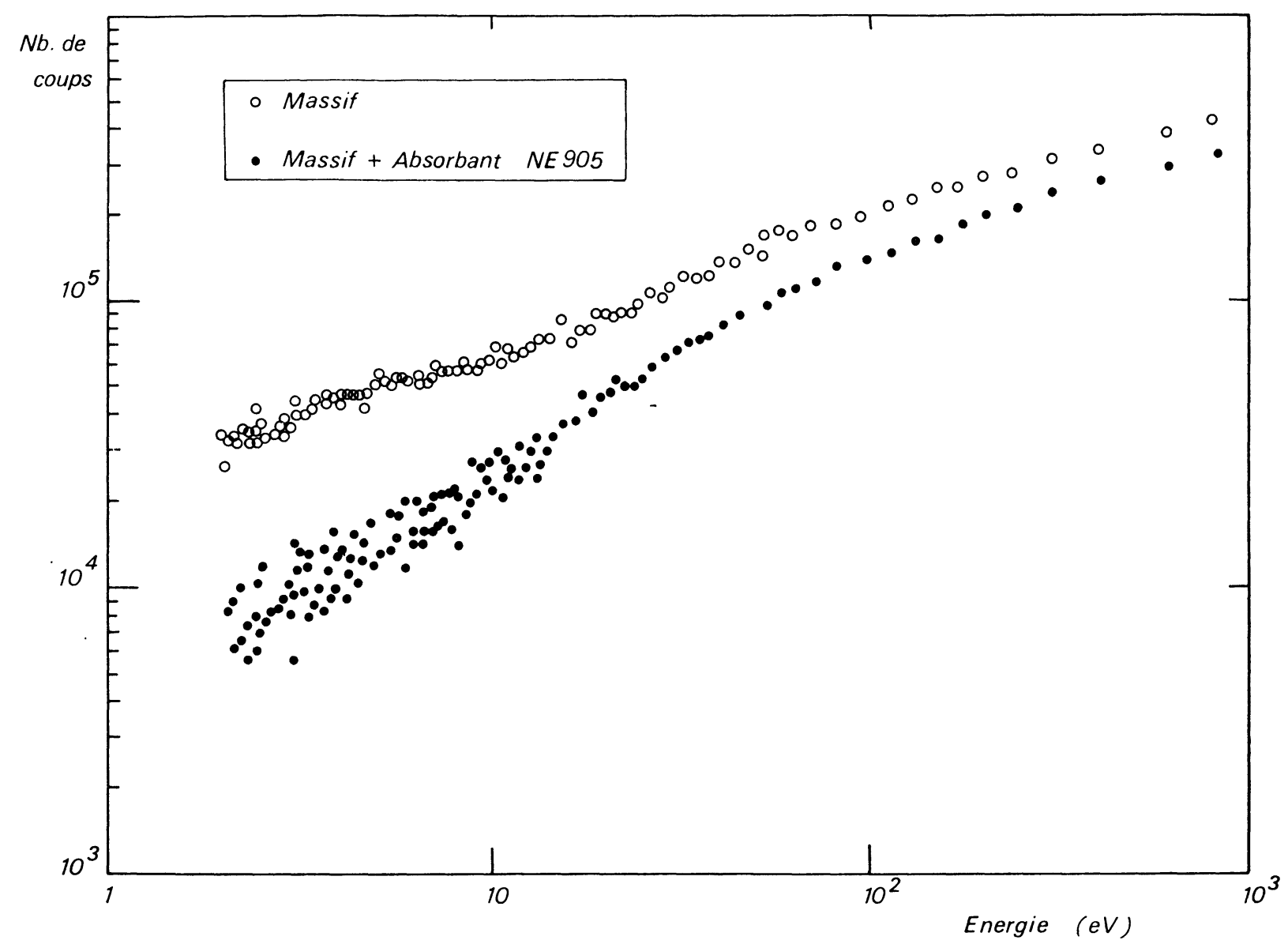

Fig. 7. - Spectre d'intercorrélation des neutrons en bout de base de vol et du code de pulsation (réponse en énergie), d'une part avec le massif, d'autre part avec le massif et un absorbant placé au milieu de la base de vol (scintillateur NE 905).

6.2 SPeCTRE DE DIfFusion. - La diffusion élastique des neutrons lents sur une cible de tungstène naturel de $50 \mu$ d'épaisseur a été étudiée à l'aide de deux

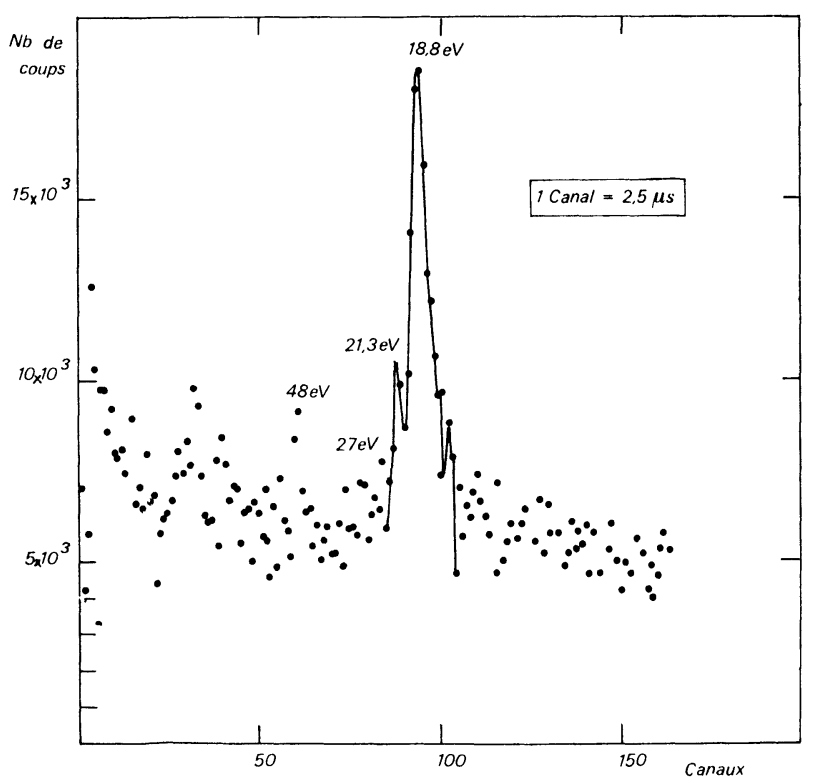

Fig. 8. - Spectre d'intercorrélation des neutrons diffusés en bout de base de vol sur du tungstène et du code de pulsation (spectre de diffusion sur le tungstène). détecteurs KG $2 \mathrm{~L}$ placés à $180^{\circ}$ l'un de l'autre autour de l'échantillon dans la protection en plomb. Le résultat obtenu (Fig. 8) est analogue à ceux qui sont publiés dans la littérature [21], [22], [23]. Le rapport bruit/signal aurait été de 250 si l'on avait utilisé la méthode classique avec le même accélérateur. La résonance de $18,8 \mathrm{eV}$ aurait été complètement noyée dans le bruit de fond. La méthode de corrélation a nécessité 20 heures de faisceau avec la fluence déjà indiquée.

7. Conclusion. - La méthode de corrélation a permis d'obtenir des résultats pour des énergies de neutrons de 3 à $50 \mathrm{eV}$, alors que la méthode classique était inapplicable essentiellement en ce qui concerne le spectre de diffusion où le rapport signal sur bruit est très faible. Cette méthode se présente comme complémentaire de la méthode classique, et a d'autant plus d'intérêt que le bruit de fond est important et que l'on s'intéresse à des parties hautes de spectre.

Signalons que les résultats obtenus peuvent être sensiblement améliorés.

1) Par l'emploi d'un flux de neutrons plus intense.

2) Par utilisation de codes plus longs afin de diminuer l'influence du bruit de fond sur la fonction de corrélation (terme en $b^{\prime} / N$ ) et de s'affranchir aussi d'une 
expérience supplémentaire de mesure de bruit. C'est, en plus de l'intérêt du calcul en temps réel et de la souplesse d'emploi, ce qui nous a poussés à construire un corrélateur digital [24] qui nous autorise à prendre des longueurs de code aussi grandes que l'on veut.

3) En utilisant des codes binaires spéciaux qui permettent de travailler avec des rapports cycliques infé- rieurs à $50 \%$. On démontre [25] qu'on peut optimaliser la méthode de corrélation et améliorer sensiblement les précisions statistiques en adaptant ce rapport cyclique à la valeur du rapport signal sur bruit de l'expérience et à la partie du spectre à étudier. On montre en particulier qu'on a intérêt à diminuer le rapport cyclique lorsque $\bar{b} / \bar{S}$ diminue.

\section{Bibliographie}

[1] Stern, T., Valet, J., Blaquière, A., Reactor Science and Technology, Parts A/B 16 (1962) 499.

[2] Rajagopal, V., Nucl. Sci. Eng. 12 (1962) 218.

[3] SKöLD, K., Nucl. Instrum. Methods 63 (1968) 114.

[4] GompF, G., Reichardt, W., Gläser, W., Beckurts, K. H., Neutron inelastic scattering, IAEA Vienne II (1968) p. 417.

[5] Prices, D. L. and SköLd, K., Nucl. Instrum. Methods 82 (1970) 208.

[6] Blanc-lapierre, A., Dumontet, P., C. R. Hebd. Séan. Acad. Sci. 250 (1960) 1216 ; C. R. Hebd. Séan. Acad. Sci. 250 (1960) 1456.

[7] VIRJo, A., Nucl. Instrum. Methods 63 (1968) 351.

[8] VoN JAN, R., SCHERM, R., Nucl. Instrum. Methods 80 (1970) 69.

[9] Rotival, M., Gerson, A., Escudié, B., Depraz, J., Avantprojet d'étude par intercorrélation des réponses des milieux en présence de neutrons. Rapport LYCEN 6825 (1968).

[10] Depraz, J., Rotival, M., Etude des milieux nucléaires par les méthodes statistiques. Communication présentée à la Rencontre des utilisateurs du Traitement du Signal, Grenoble, 16 décembre 1968.

[11] Billerey, R., Charnay, D., Depraz, J., Descroix, E., LYCEN 7262 (1972).

[12] Rotival, M., Thèse Doct. spécialité, Univ. de Lyon (avril 1970).
[13] Peterson, W. W., Error correcting codes (John Wiley and Sons N. Y. ) 1961.

[14] Solomon W. Golomb, Shift Register Sequences (HoldenDay Inc.) 1967.

[15] Depraz, J., Gerson, A., Rotival, M., Escudié, B., LYCEN 6875 (1968).

[16] Michaudon, A., Reactor Science and Technology 13 (1963) 165.

[17] Firk, F., Slaughter, G., Ginther, R., Nucl. Instrum. Methods 13 (1961) 313.

[18] Bollinger, L. M., Thomas, G. E., Nucl. Instrum. Methods 17 (1962) 97.

[19] Jung, H., Priesmeyer, G., Nucl. Instrum. Methods 68 (1969) 353.

[20] Sowerby, M. G., PAtrick, B. H. et al., J. Nucl. Energy 24 (1970) 323.

[21] Hughes, D. J. et HaRvey, J. A., BNL-325.

[22] Garnsworthy, R. K., Broomhall, G. J., Nucl. Instrum. Methods 57 (1967) 82.

[23] King, T. J., Fullwood, R. R., Block, R. C., Nucl. Instrum. Methods 52 (1967) 321.

[24] Charnay, D., Chevalier, H., Gorius, J. P., Rapport annuel Institut de Physique Nucléaire de Lyon, LYCEN 7301 (1973) 52.

[25] Hossfeld, F., Amadori, R., Scherm, R., Instrumentation for neutron inelastic scattering research, IAEA Vienne (1970) 117. 